\title{
PWE-072 PROSPECTIVE COMPARISON OF DIFFERENT GASTRIC MOTILITY TESTS IN DYSPEPTIC PATIENTS
}

\author{
doi:10.1136/gut.2011.239301.335
}

MFJaboli, 1,* JBell, ${ }^{2}$ CClayman, ${ }^{1}$ JBuscombe, ${ }^{2}$ LMarelli, ${ }^{1}$ OEpstein ${ }^{1}{ }^{1}$ Gastroenterology, Royal Free Hospital, London, UK; ${ }^{2}$ Radiology and Nuclear Medicine, Royal Free Hospital, London, UK

Introduction Up to $60 \%$ of dyspeptic patients have a normal upper endoscopy. Endoscopy is unable to diagnose myoelectrical disorders which can cause dyspeptic symptoms including epigastric discomfort, early satiety, bloating, nausea and vomiting. Although patients with functional dyspepsia complain of epigastric symptoms, the relation between these symptoms and gastric motility remains controversial. Electrogastrography (EGG) is a simple, non-invasive method for assessing the gastric pacemaker slow wave. Gastric emptying study (GES) is typically the first test in evaluation of gastric motor function using a radiolabelled test meal. The ultrasound of the stomach (USS) is a recent non-invasive and cheap modality to evaluate gastroduodenal motility. We wanted to compare the information given by three different gastric motility tests in patients with endoscopy negative dyspepsia (END).

Methods A series of 77 dyspeptic helicobacter pylori negative patients (60 female: 17 male, ages 18-65 years) diagnosed according to the Roman III criteria and a normal upper endoscopy, underwent a standard fasting and postprandial EGG, a technetium labelled mashed potato GES and an ultrasound of the stomach given a soup test meal. The EGG was considered abnormal if $<70 \%$ of slow wave activity was recorded at 3 cycles/min. Delayed or increased gastric emptying was diagnosed if the $T^{1} 1 / 2$ for the test meal was outside the normal

range for our unit ( $<15$ or $>45 \mathrm{~min}$, respectively). The USS was considered normal if the gastric emptying rate was between $45 \%$ and $78 \%$.

Results Thirty-one (40\%) patients had both GES and USS test showing gastroparesis with mainly symptoms of nausea and vomiting (20/31,64\%). Eighty per cent (25/31) of these patients had an abnormal EGG, having as a predominant pattern an abnormal pre and post prandial test (12/31,38\%). Interestingly 17 people $(22 \%)$ had normal GES and USS, with abnormal EGG in $82 \%$ of patients (14/17). Ten of these patients $(58 \%)$ were experiencing nausea and vomiting as well. Only $2(2.5 \%)$ patients had findings of dumping syndrome on GES and USS. There was more heterogeneity in the results of these tests with patients referring bloating and epigastric discomfort.

Conclusion In patients with endoscopy negative dyspepsia, gastroparesis is very common and particularly with symptoms like nausea and vomiting. GES and USS can provide a firm diagnosis and possibly influence treatment strategies. We think these investigations should be incorporated into dyspepsia guidelines at the point where endoscopy is reported as normal. We are currently investigating the role of EGG for the poor correlation with the other tests.

Competing interests None.

Keywords dyspepsia, gastric motility. 